\title{
Effects of directional and neu tral category labels in bidimensional rule-learning problems
}

\author{
PAUL G. NEUMANN \\ University of Colorado, Boulder, Colorado 80302
}

\begin{abstract}
In bidimensional concept problems, the distinction between primary and complementary rule forms is, in some sense, a function of the directional nature of the sorting category labels. Primary rules are those rules which assign the TT class to the "positive" category. Complementary rule forms are obtained by reversing these category assignments. Instead of directional labels, neutral labels can be used. Reversing these labels creates no fundamental change in the solution of the problem. The present experiment investigates the effect of directional and neutral category labels in a design which incorporates all 12 problems defined by the combination of directional and neutral labels. Significant effects of labels on performance were obtained. Implications of these effects for hypotheses advanced by Seggie (1969), Peters and Denny (1971), and Bourne and Guy (1968) are discussed.
\end{abstract}

Bourne (1970) has shown that the calculus of propositions defines eight unique, nontrivial rules which can be used to generate bidimensional concepts. These rules act as logical operators on combinations of two or more stimulus attributes to produce a partitioning of the stimulus population. Each attribute may be present or absent in a given stimulus configuration. When only two attributes are relevant, there are four state permutations: present-present, present-absent, absent-present, and absent-absent. It has become conventional to apply the logical truth table notation true-true (TT), true-false (TF), false-true (FT), and false-false (FF) to these states.

An examination of the eight bidimensional rules reveals that they define only four unique partitionings of the stimulus population. The distinction between the four "primary" rules and the four "complementary" rules lies in the arbitrary designation of that stimulus subset which contains the TT class as being "correct" or "positive" in the case of primary rules and that stimulus subset which does not contain the TT class as being "correct" or "positive" in the case of complementary rules. The distinction is thus a function of the labels which are assigned to the subsets. In the laboratory, this function manifests itself in the choice of labels for the sorting categories, which are isomorphic to the stimulus subsets. Typical labels are positive/negative, plus/minus, $y e s / n o$, and $V E C / n o t$ a $V E C$. These labels, and others

\footnotetext{
*This article is based on a thesis submitted in partial fulfillment of the requirements for the MA degree at the University of Colorado. The study was supported by Grant GB $34077 \mathrm{X}$ to the Institute for the Study of Intellectual Behavior and by Grant GJ 453 to the Computer Laboratory for Instruction in Psychological Research, both from the National Science Foundation; and also by Grant MH 14314 to Lyle E. Bourne, Jr. from the National Institute of Mental Health and by an NDEA Graduate Fellowship awarded to the author, who wishes to express his gratitude for generous support and helpful criticism offered by Lyle E. Bourne, Jr., William F. Battig, and Peter G. Polson. This is Publication No. 49 of the Institute for the Study of Intellectual Behavior. Requests for reprints should be sent to Paul G. Neumann, Institute for the Study of Intellectual Behavior, University of Colorado, Boulder, Colorado 80302.
}

which have a strong natural ordering, we shall call "directional labels."

Instead of directional labels, it is possible to apply labels with no natural ordering to the sorting categories. We shall call these labels "neutral" in that they do not tend to direct S's attention differentially or connote differential values between the two sorting categories.

Neutral labels render the distinction between primary and complementary labels meaningless. There is no longer a "positive" and a "negative" sorting category. Reversing the labels creates no fundamental change in the nature of the solution of the concept.

Suppose, for example, that the stimulus set consists of all permutations of two dimensions and three values. This creates nine unique stimulus patterns $\left(3^{2}\right)$. The

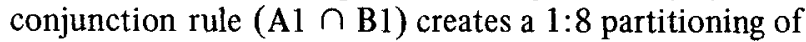
the stimulus set. That is, of the nine unordered combinations, only one satisfies the conjunction rule requiring the simultaneous presence of both relevant values, one from each dimension. Thus, only one combination of values "is" the concept. Its complement, the alternative denial rule $(\overline{\mathrm{A} 1} \cup \overline{\mathrm{B} 1})$ creates the same partitioning. However, there are now eight stimulus combinations which satisfy the rule requiring the absence of one or both relevant values. Thus there are eight stimuli which "are" the concept.

Instead of defining what "is" and what "is not" the concept, the two subsets may be labeled with unique, unordered labels. Ss may then choose to solve for either subset by identifying the stimulus combinations which belong to that subset and assigning the rest to the other subset by default (category focusing). Using a combination of directional and neutral labels results in 12 rule forms as illustrated in Table 1.

To simplify further reference to the 12 problems, the following notation will be used. Roman numerals will refer to the partitions of the stimulus classes. Roman numeral $\mathrm{I}$ is assigned to the conjunction/neutral/alternative denial partition, numeral II to the 
Table 1

The Twelve Rule Forms

\begin{tabular}{lcccc}
\hline \multirow{5}{*}{ Partition I } & Class & Primary & Neutral & $\begin{array}{c}\text { Comple- } \\
\text { mentary }\end{array}$ \\
\hline \multirow{5}{*}{ Partition II } & TT & + & $\Phi$ & - \\
& TF & - & $\theta$ & + \\
& FT & - & $\theta$ & + \\
& FF & - & $\theta$ & + \\
& TT & + & $\Phi$ & - \\
\multirow{5}{*}{ Partition III } & TF & + & $\Phi$ & - \\
& FT & + & $\Phi$ & - \\
& FF & - & $\theta$ & + \\
& TT & + & $\Phi$ & - \\
Partition IV & TF & - & $\theta$ & + \\
& FT & + & $\Phi$ & - \\
& FF & + & $\Phi$ & - \\
& TT & + & $\Phi$ & - \\
& TF & - & $\theta$ & + \\
& FT & - & $\theta$ & + \\
\hline
\end{tabular}

disjunction/neutral/joint denial partition, numeral III to the conditional/neutral/exclusion partition. This order of partitions reflects increasing difficulty of primary rule problems as established by previous research (cf. Bourne, 1970). The letters $\mathrm{P}, \mathrm{N}$, and $\mathrm{C}$ will refer to the primary, neutral, and complementary forms of the rule within partitions. Thus $\mathrm{I}(\mathrm{P})$ refers to the conjunction rule, III(C) refers to the exclusion rule, etc.

Seggie (1969), Peters and Denny (1971), and Gottwald (1971) have demonstrated that significant differences in performance can be obtained in the solution of selected bidimensional concept problems between conditions in which the labels of the response categories are directional and conditions in which the labels are neutral. Peters and Denny (1971) utilized a rule-learning task in which the directional labels were yes and $n o$ and the neutral labels were $A$ and $B$, despite the fact that $A$ and $B$ have a strong natural ordering. The design also incorporated a procedure by which $\mathrm{S}$ was required to write the current hypothesis following each trial, and $\mathrm{E}$ corrected $\mathrm{S}$ if the hypothesis was not consistent with the relevant values. Some indication that the results are anomalous is evidenced by the fact that in the directional label conditions, performance was better for the biconditional rule problem than for the conditional rule problem. Under these experimental conditions, the opposite ordering is well documented.

Peters and Denny accounted for their results by assuming that in the directional label conditions, $S$ focuses on the positive sorting category. Since the conditional rule, III(P), assigns three of the four classes to the positive category, it was assumed that the solution of the problem would be easier for the biconditional rule, which assigns only two classes to the positive category. The neutral label problems, III(N) and IV(N), were ordered in the opposite manner. This was accounted for by assuming that the sorting category containing the fewest truth table classes would be the focus category in the neutral label problems. Thus III(N), with only one class in the focus category, should be easier than IV(N), with two classes in each category. These assumptions may be extended to produce a qualitative prediction for the distribution of all 12 problems previously defined. This distribution is illustrated in Fig. 1a.

The results reported by Seggie (1969), who found that $\mathrm{I}(\mathrm{P})$ was easier than $\mathrm{II}(\mathrm{P}), \mathrm{I}(\mathrm{N})$, and $\mathrm{II}(\mathrm{N})$, were accounted for in terms of the explicitness of the task demands, involving different "levels of learning." Specifically, it was hypothesized that the conjunction rule, I(P), demands learning only that the TT class is assigned to the positive sorting category, whereas neutral label problems and more complex directional label problems demand learning of all classifications. The problems are thus hypothesized as demanding two different levels of learning. Presumably, this rationale holds for all problems which are perceived as more complex than the conjunction rule problem. Since the higher "level of learning" demands learning all classifications, this would seem to place an upper limit on the difficulty of solving bidimensional problems, resulting in the qualitative distribution illustrated in Fig. $1 \mathrm{~b}$.

Gottwald (1971) utilized positive and negative as directional labels which, like $A$ and $B$, have a strong natural ordering. In addition, the stimulus population was composed of only two values per dimension, which, as the author notes, permits solution of a single problem with more than one rule. For example, Partition
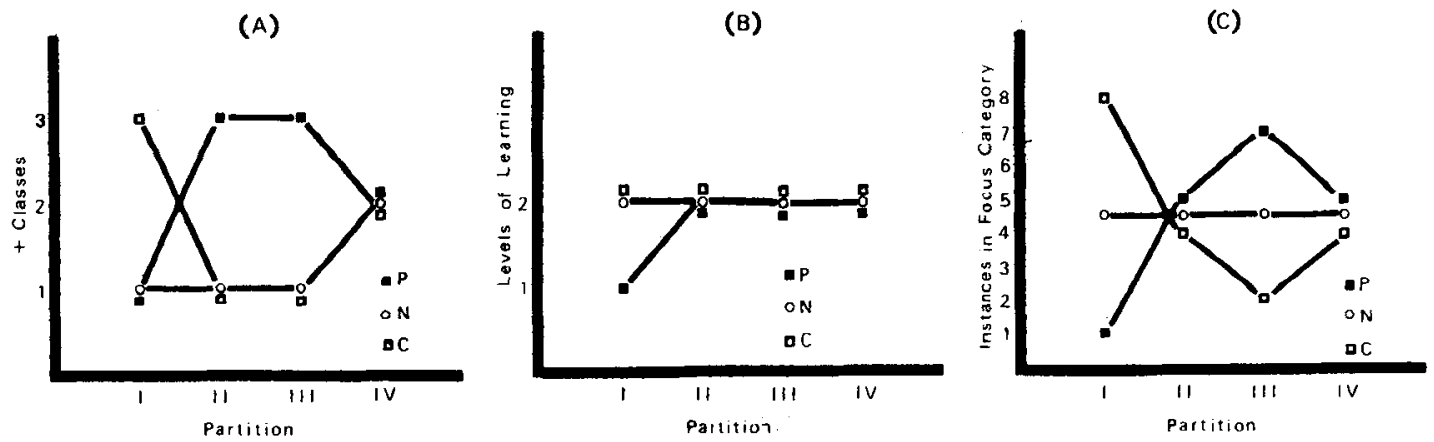

Fig. 1. Qualitative distributions derived from the hypotheses of Peters and Denny (1971), Seggie (1969), and Bourne and Guy (1968). Partitions are ordered by increasing difficulty of primary-rule problems. 
contains $\mathrm{A} 1 \mathrm{~B} 1$. in one subset and $\mathrm{A} 1 \mathrm{~B} 2, \mathrm{~A} 2 \mathrm{~B} 1$, and $\mathrm{A} 2 \mathrm{~B} 2$ in the other. When the positive label is assigned to the A1B1 subset, the problem can be solved with either the conjunction rule $(\mathrm{A} 1 \cap \mathrm{B} 1)$ or the joint denial rule $(\overline{\mathrm{A} 2} \cap \overline{\mathrm{B} 2})$. When the positive label is assigned to the $\mathrm{A} 1 \mathrm{~B} 2, \mathrm{~A} 2 \mathrm{~B} 1, \mathrm{~A} 2 \mathrm{~B} 2$ subset, the problem can be solved with either the disjunction rule $(A 2 \cup B 2)$ or the alternative denial rule $(\overline{\mathrm{A} 1} \cup \overline{\mathrm{B} 1})$. Studies have shown that not only do different rules vary in difficulty, but also the difficulty of using information in the four truth table classes varies across rules (cf. Haygood \& Bourne, 1965). Since Gottwald's design is such that any problem may be solved with more than one rule, the data must be considered uninterpretable.

Predictions for a third qualitative distribution may be derived from the category homogeneity hypothesis of Bourne and Guy (1968). Their results supported the hypothesis that in a rule-learning problem, difficulty across problems increases with the number of instances assigned to the positive sorting category. Although Bourne and Guy did not specifically address themselves to the problem of neutral labels, if the term focus category is substituted for positive category, it may be assumed that choice of a focus category is essentially random in the neutral label problems, producing a condition in which, within each partition, approximately half the Ss will solve an analog of the primary rule and half an analog of the complementary rule. Thus, within each partition, performance on the neu tral label problem should lie approximately midway between performance on the two directional label problems. This rationale produces the qualitative distribution illustrated in Fig. 1c.

In addition to providing a test of the above hypotheses, a design which includes the neutral label problem in all four partitions of the stimulus population permits the assessment of the difference in performance across problems which differ only in the manner in which the truth table classes are partitioned into two response categories, independent of any interaction between the coding of instances and the coding of response category labels. For example, it might reasonably be hypothesized that the coding of an FF class stimulus would reflect negation of the presence of the relevant attributes. Additional difficulty in learning the assignment of such negatively coded stimuli might be induced by the necessity of assigning such stimuli to a category coded positively, and, similarly, positively coded stimuli might be more difficult to learn when assigned to a negatively coded category. The neutral label problems eliminate this possible interaction. Differences in performance across neutral label problems must, in some manner, derive solely from the partitioning of the stimulus population.

\section{METHOD}

Design

The basic design is a 4 by 3 by 2 factorial. There are three rule forms (primary, neutral, complementary) in each of the four partitionings of the stimulus population. In addition, there are two stimulus populations, each based upon a different set of relevant values. A rule-learning reception paradigm was employed. Each S solved a single problem, the solution of which was based upon one of the 12 rule forms illustrated in Table 1.

\section{Subjects}

Ss were 150 undergraduate psychology students who volunteered for the experiment in partial fulfillment of optional course requirements. Their assignment to the 24 experimental conditions was based upon the order in which their names appeared on sign-up sheets.

\section{Materials}

Geometric stimuli varied on the dimensions size (large, medium, small), color (red, yellow, black), and shape (triangle, square, hexagon) for the population in which large and red were the relevant values and color (red, yellow, black), shape (triangle, square, hexagon), and number $(1,2,3)$ for the population in which 2 and square were the relevant values. The populations were constructed in such a manner that there were equal numbers of stimuli in all truth table classes. Actual stimuli consisted of colored $35-\mathrm{mm}$ slides rear-projected on a ground glass screen.

Two sets of category labels were used. The first set consisted of the CVC trigram $V E C$ for the "positive" category and not a $V E C$ for the "negative" category. The neutral labels were $V E C$ and $X A D$. The second set consisted of $D A X$ and not a $D A X$ for the directional labels and $D A X$ and $C E V$ for the neutral labels.

Scoring protocols were generated by means of a FORTRAN IV program. The program automatically alternated label sets and relevant values across replications of the basic experiment consisting of 12 conditions. In addition, the program generated a unique random sequence of stimuli for each of the replications.

\section{Procedure}

Upon entering the laboratory, $\mathrm{S}$ was seated in front of the projection screen. Instructions were read, and $E$ ensured that $S$ understood the instructions, reinstructing from the typewritten instructions or by analogy when necessary. Ss were free to ask questions, and $\mathrm{E}$ answered those questions which did not demand information beyond that included in the instructions.

Instructions defined the term "concept" and incorporated truth table training based upon playing card dimensions red/black and face card/number card. The four truth table classes were shown partitioned in a $1: 3$ and $2: 2$ ratio. Ss were told that their task was to view a series of slides, each of which was capable of being classified into one or two categories, and to learn to make these classifications correctly. It was asserted that a rule governing the classification could be learned, and a rule which reduces to the affirmation rule (e.g., red is positive) was used as an illustration. The relevant values and sorting category labels were typed on standard $3 \times 5$ white notecards and were displayed at the appropriate point in the instruction phase. They remained visible to $S$ throughout the experiment.

Stimuli were presented in a predetermined random order. No limit on study time was imposed. Slides remained visible to $S$ approximately $5 \mathrm{sec}$ after feedback was given, after which the projector was advanced to the next slide. A learning criterion of 16 consecutive correct responses was imposed. Ss reaching criterion numbered 144. Five Ss were terminated as nonlearners on the first error following the 79 th trial: in this manner, one $S$ in the $I(P)$ condition, two $S$ s in the IV(N) condition, one $S$ in the IV(C) condition, and one $S$ in the IV(P) condition. One additional $S$ was terminated due to an $E$ error.

\section{RESULTS}

Trials to last error were analyzed for normality of the 


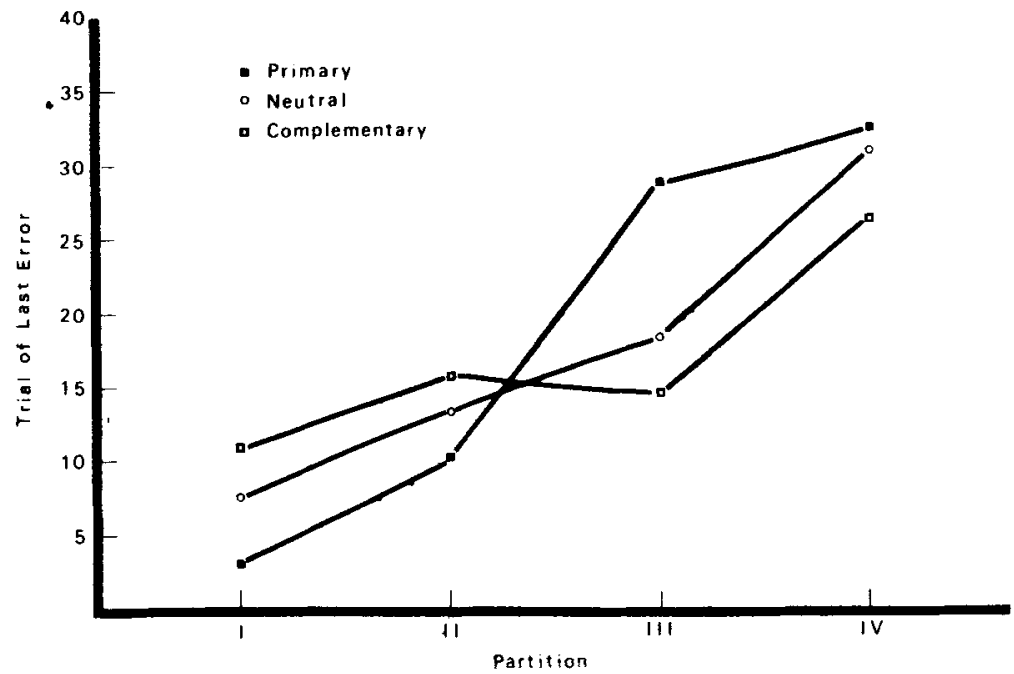

distribution and the homogeneity of variance. The raw data were found to be normally distributed, $\chi^{2}(6)=$ $6.25, p<.40$, but the hypothesis of homogeneity of variance was rejected, $F(23,5625)=2.56, p<.02$. Accordingly, the transformation $X^{\prime}=\sqrt{\mathrm{X}+1}$ was performed. This transformation satisfied both the test for normality of the distribution, $\chi^{2}(6)=10.50$, $\mathrm{p}<.10$, and that for homogeneity of variance, $\mathrm{F}(23,5625)=1.11, \mathrm{p}<.33$.

An analysis of variance was performed on both the raw scores and transformed scores. Since the results were nearly identical, all references will be to results obtained with the raw scores. Significant differences were obtained for partition, $F(3,120)=26.93, p<.01$, and the Partition by Label interaction, $F(6,120)=2.69$, $p<.02$. The significant effect of partition indicates clearly that the difficulty of the problem differs as a function of the manner in which the rules partition the stimulus population.

Independent of any labeling effects, Partition I (see Table 1) produces problems which are easiest to solve $\left(\overline{\mathrm{X}}_{\mathrm{I}}=7.19\right)$, followed by Partition II $\left(\overline{\mathrm{X}}_{\mathrm{II}}=13.19\right)$, Partition III $\left(\overline{\mathrm{X}}_{\mathrm{III}}=20.67\right)$, and Partition IV $\left(\overline{\mathrm{X}}_{\mathrm{IV}}=\right.$ 29.97). As Fig. 2 illustrates, the significant interaction between partition and label is due to the fact that in Partitions I and II, the directional labels in the primary rule configuration [I(P) and II(P)] are easier than in the complementary configuration $[\mathrm{I}(\mathrm{C})$ and $\mathrm{II}(\mathrm{C})]$, whereas in Partitions III and IV, the opposite result obtains [III(C) easier than III(P) and IV(C) easier than IV(P)]. In all partitions, the neutral label problems are approximately midway in difficulty between the means of the primary rule problems and the complementary rule problems. This last result supports the assumption that in the neutral label problems, selection of a focus category is random, and that half the Ss solve analogs of the primary and complementary rule problems. Labels were not statistically significant, $F(2,120)=.74$, nor were relevant values, $F(1,120)=.52$, Partition by Relevant Values interaction, $F(2,120)=.90$, or the three-way interaction, $F(6,120)=.80$.
Fig. 2. Mean trial of last error for the $\mathbf{1 2}$ problems. The points plotted represent raw score means collapsed across relevant-value conditions. Standard deviations in primary, neutral, and complementary order are as follows: 4.11, 4.61, and 5.12 for Partition I; 4.44, 9.61, and 13.31 for Partition II; 12.81, 13.85, and 8.99 for Partition III; and 15.36, 12.78, and 11.92 for Partition IV.
The results indicate that mean performance on problems in which the response categories are labeled neutrally lies uniformly between the mean performance on problems in which the categories are directionally labeled, supporting the hypothesis that the immediate effect of neutral labels is to produce a problem in which the choice of a focus category is random. Thus, within each partition, half the Ss in the neutral label problem solve an analog of the primary rule problem and half an analog of the complementary rule problem.

Peters and Denny (1971) hypothesized that the effect of neutral labeling was such that $S$ focuses on the category containing the fewest classes of stimuli, whereas with directional labels, $S$ assumes that the assignments to the positive category must be learned. The prediction must therefore be that $\mathrm{I}(\mathrm{N}), \mathrm{II}(\mathrm{N})$, and III(N), each of which incorporate a sorting category to which only one truth table class is assigned, should be equal in difficulty. Figure 2 illustrates that this is clearly not the case.

The difficulty of the neutral label problems increases across partitions. Further, this hypothesis predicts that performance on all directional label problems with the same number of classes assigned to the positive category should be equal, and presumably that performance should decrease with an increasing number of classes so assigned. Comparison of performance on II(P), which has three classes assigned to the positive category, with that of IV(P), which has two classes assigned to the positive category, again indicates that this hypothesis is not viable. Performance on $\mathrm{II}(\mathrm{P})(\overline{\mathrm{X}}=10.33, \mathrm{SD}=4.44)$ is better than on $\operatorname{IV}(\mathrm{P})(\overline{\mathrm{X}}=32.42, \mathrm{SD}=15.36)$.

Seggie (1969) concluded that his results could be accounted for in terms of two levels of learning demanded by the problems. The conjunction rule, I(P), was hypothesized to require a lower level of learning than other rule forms, which require that inferences be made and that the assignment of all stimuli be learned. The results indicate that, whereas this hypothesis is 
capable of generating adequate qualitative predictions as to performance on the primary and neutral label problems in Partition I and Partition II, it fails to generalize to Partition III and Partition IV. All problems in these partitions are predicted to be equal in difficulty. Figure 2 illustrates that the results are not congruent with this prediction.

An extension of the hypotheses of Bourne and Guy (1968) results in the prediction that performance on neutral label problems should lie midway between performance on the directional label problems, since choice of a focus category is essentially random. Thus it is predicted that half the Ss solve analogs of each of the two directional label problems. Figure 2 indicates that this prediction is supported. However, there are at least two deficiencies in this approach. First, based upon the number of instances assigned to the positive category, which is assumed to be the focus category in directional label problems, performance on II(C) should be better than performance on II(P). The opposite ordering obtains. A second deficiency is that since Partition I incorporates the most disparate partitioning, it must be predicted that the greatest within-partition difference in performance would lie in this partition. The results indicate that the greatest differences lie in Partition III. This result suggests that if, in fact, Ss begin the problem with a conjunctive set, there also exists a mechanism by which all assignments defined by this set may be quickly negated, perhaps by simply reversing the category label assignments when consistently negative feedback is present in the initial trials.

The significant differences across partitions are at least partially accounted for by the conclusion of Bourne and Guy that Ss tend to treat TF and FT classes as a single type of instance which incorporates one relevant attribute but not the other, and that it is difficult for Ss to learn to assign TT and FF class stimuli to the same response category. Thus, partitions which assign TF and FT to different categories and partitions which assign TT and FF to the same category should be more difficult. However, since Partition III incorporates both of these conditions and Partition IV only incorporates the latter, the relatively poorer performance in Partition IV is unexplained.

The differences in performance on neutral label problems indicate that independent of interaction between the coding of the instances and the coding of the response category labels, the manner in which the stimuli are partitioned is a variable affecting performance in rule-learning problems. It should be noted that this effect cannot be characterized as a "linear trend," since the order of partitions is arbitrarily based upon the previously obtained order of difficulty of the primary rule problems. Once again, this effect supports the conclusions of Bourne and Guy that TF and FT classes are treated as a single class and that it is difficult to learn to assign TT and FF classes to the same response category, since these factors are solely dependent upon the manner in which the rules partition the stimulus classes, independent of the labels assigned to the response categories.

Quite independent of theoretical considerations is the empirical demonstration that the directional quality of the category labels affects performance and that, since there is significant interaction between labels and partitions, this variable must be considered when assessing results in rule-learning experiments, especially when results across experiments are being compared. Consider, for example, two hypothetical experiments, both of which purport to compare performance in $\mathrm{II}(\mathrm{C})$ and III(C) but one of which incorporates directional labels and the other neutral labels. Opposite orders of difficulty will obtain. The most marked effect, however, would obtain in experiments incorporating both primary and complementary rule forms. Such experiments, including those involving complemental shifts, should be evaluated carefully in the light of the present empirical findings. ${ }^{1}$

\section{REFERENCES}

Bourne, L. E., Jr. Knowing and using concepts. Psychological Review, $1970,77,546-556$.

Bourne, L. E., Jr., \& Guy, D. E. Learning conceptual rules. II: The role of positive and negative instances. Journal of Experimental Psychology, 1968, 77, 488-494.

Gottwald, R. L. Effects of response labels in concept attainment. Journal of Experimental Psychology, 1971, 91, 30-33.

Haygood, R. C., \& Bourne, L. E., Jr. Attribute- and rule-learning aspects of conceptual behavior. Psychological Review, 1965 , 72, 175-195.

Peters, K. G., \& Denny, J. P. Labeling and memory effects on categorizing and hypothesizing behavior for biconditional and conditional conceptual rules. Journal of Experimental Psychology, 1971, 87, 229-233.

Seggie, J. L. Levels of learning involved in conjunctive and disjunctive concepts. Australian Journal of Psychology, 1969, 21. 325-333.

\section{NOTE}

1. A list of 22 sets of category labels have been scaled for "oppositeness" and "difference." A table of the results is available from the author upon request.

(Received for publication December 17, 1973; revision received March 22, 1974.) 